\title{
On Probability of Surf-Riding in Irregular Seas with A Split-Time Formulation
}

\author{
Vadim Belenky ${ }^{1}$, Kenneth Weem ${ }^{1}$ s, Kostas Spyrou ${ }^{2}$ \\ ${ }^{1}$ David Taylor Model Basin - NSWCCD, 9500 Macarthur Blvd., W. Bethesda, MD, USA. \\ ${ }^{2}$ National Technical University of Athens, 9 Iroon Polytechneiou, Zographos, 15780, Greece
}

The paper reviews the status of a multi-year research effort for using the split-time method to calculate the probability of ship capsizing due to broaching-to in irregular waves. This required taking steps toward extending the existing theory of surf-riding and broaching-to, from regular waves to irregular waves and applying it to numerical simulation codes of ship motions. The extension of the theory for irregular waves leads to the formulation of a spatial-temporal framework for considering surf-riding where the celerity of irregular waves must be defined. An approximate metric for the likelihood of surf-riding in irregular waves has been proposed as the distance, in the phase plane, between the instantaneous position of a ship and the stable surfriding quasi-equilibrium at that instant. Further work includes studying the properties of the surfriding phase plane in irregular waves and statistics of surf-riding occurrences.

Keywords: Probability, Surf-Riding, Celerity, Split-Time Method

\section{INTRODUCTION}

Surf-riding and broaching-to are known to be associated, complex phenomena, linked through the generation of attraction towards a point of equilibrium ("quasi-equlibrium" for an irregular sea) located near a wave trough, appearing to be stable in surge but unstable in yaw. This yaw instability leads to a rapid, uncontrollable turn which can induce a large roll angle or capsize. The standing theory of surf-riding and broaching-to was proposed on the basis of the nonlinear dynamics theory (Spyrou 1996, 1997). It used an ordinary differential equations (ODE) model of ship motion in regular following/quartering waves. The minimal mathematical model for describing these phenomena includes surge-sway-yaw for modeling broaching-to, plus a roll motion equation to model capsize. Rudder control must also be included for the directional control of the ship. The resulting system has a 10-dimensional phase space whose rigorous investigation poses a very challenging task.

This already complex setup becomes even more complex when, the ship motions are calculated with high accuracy, by using the more consistent, in ship hydrodynamics terms, formulation. Wave excitation can no longer be separated from stiffness while radiation and diffraction forces add a hydrodynamic memory effect. Furthermore, the irregularity of realistic ocean introduces new physical qualities to the phenomenon.

The challenge to include surf-riding and broaching-to into a probabilistic assessment of stability based on advanced hydrodynamic codes has been taken up by the US Office of Naval Research (ONR) project "A Probabilistic Procedure for Evaluating the Dynamic Stability and 
Capsizing of Naval Vessels". The project's main objective is to create a robust theory of probabilistic capsizing in irregular waves and a numerical procedure based on this theory.

The split-time method is the probabilistic framework of this project. Like all other methods that use the separation of dynamics in order to attack a complex problem, the idea of the splittime method is to divide the problem of probability calculation of a rare, extreme event, in two problems that may be easier to handle. The "non-rare" problem is associated with an intermediate random event, statistics of which can be obtained by running an advanced code for sufficient time. This "non-rare" problem ensures a correct relation between time and probability in irregular waves. The "rare" problem is responsible for modeling the physics of the rare event. Its core is a metric of the danger of the severe event, for example capsizing or large roll angle caused by broaching-to, that is computed when the intermediate random event occurs. The procedure is repeated for each intermediate random event observed during the non-rare simulations. The sample of metric values is then statistically extrapolated to determine the likelihood of the rare event. Since the metric value is expected to include the physics of the extreme event, actual observations of the event are not required, so the method is expected to work for conditions (sea states, speed, loading) where extreme events are rare.

Before presenting details on the devised metric for surf-riding's probability calculation, we shall provide an exposition of relevant progress achieved recently, covering the reproduction of the aforementioned phenomena with an established hydrodynamic code, the calculation of celerity for irregular seas and the spatio-temporal formulation of surf-riding and broaching-to for such an environment.

\section{SURF-RIDING AND BROACHING-TO PRODUCED BY THE HYDRODYNAMIC CODE LAMP}

\subsection{Objective and Approach}

As the current theory of surf-riding and broaching-to was developed through a mathematical model involving ordinary differential equations (ODE) only, its relevance to more general models raised certain questions. Should ship motions be calculated by pressure integration, with 6 degrees of freedom and hydrodynamic memory effects, can the current theory still capture the essential aspects of system's extreme dynamics? How well will the analysis tools of nonlinear dynamics work with the dynamical system represented by the advanced hydrodynamic code? The results of a study of these issues are presented in Spyrou et al. (2009) and Belenky et al. (2010).

While the Large-Amplitude Motion program (LAMP) was used as the main hydrodynamic code for this study, the results are meant to be code-independent and applicable to any code capable of reproducing surf-riding, broaching-to, and capsizing. LAMP is a based on a boundary value problem for radiation and diffraction (potential flow), a body non-linear evaluation of the hydrostatic and Froude-Krylov forces, and force models for vortex and viscous effects (Lin and Yue 1990). The use of LAMP for the simulation of ship maneuvering is described in Lin et al. (2006) and Yen et al. (2010). 
It is understood that simulation codes based on volume-grid viscous flow solvers can provide a more complete and, in many ways, advanced solution than LAMP, which must resort to derivative models for viscous and vertical effects. However, the extraordinary computation cost of such simulations precludes their application to any large scale evaluation of ship motions in irregular seas. Hybrid codes such as LAMP can provide realistic time-domain simulations of ship motion incorporating the key physical effects of large amplitude vertical motion coupled with unsteady lateral motions associated with surf-riding and broaching-to, and are likely to be the principal tool for long-duration nonlinear seakeeping analysis in the near future. As simulations with more advanced codes become practical, the approach and procedures of the split-time method will be directly applicable to those results as well.

The ship configuration used for demonstrating the surf-riding and broaching-to phenomena is the "tumblehome" form of the ONR Topsides Study (Bishop et al. 2005).

\subsection{Time-Domain Simulations}

The study first looked at the modeling of large heel angle (up to capsize) caused by a sharp turn. Figure 1 shows predicted time histories of roll with two values of the transverse metacentric height (GM) after a $30^{\circ}$ rudder application while sailing at 38 knots in calm water. At the higher GM value, the ship attains a large heel angle before recovering. At the lower GM value, the ship capsizes.

The study then looked at surf-riding in combinations of wave frequency and height and ship speed for which the theory predicts a co-existence of surging and surf-riding. Figure 2 shows different responses depending on the initial conditions. Systematic simulations were performed for a nominal Froude number $(F n)$ from 0.3 to 0.41 and commanded headings relative to the wave direction from 10 to 32 degrees. A summary of the results is presented in Figure 3.

A remarkable feature that appeared at the higher nominal speeds $(F n>0.33)$ was a stable oscillatory type of surf-riding, for which the time history of ship speed is shown in Figure 4. As the ship is carried along by a single wave, it is also oscillating up-and-down the wave face. This fascinating occurrence has been observed in the past and has been explained as being due to a Hopf bifurcation (Spyrou 1996).

\subsection{Application of "Continuation Method"}

"Continuation" is a powerful approach for studying the behavior of nonlinear systems, by mapping and characterizing steady states. The first use of a continuation method (DERPAR) with a potential flow code (LAMP) was described by Spyrou et al. (2009). To avoid excessive complexity associated with the memory effect, the diffraction and radiation forces have been approximated with constant added mass and damping coefficient.

A sample result from LAMP-based continuation analysis is given in Figure 5. The curve shows the positions of surf-riding equilibria in the coordinates as yaw vs. rudder angle, and follows closely the similar curve from Spyrou (1996). This curve contains both stable and unstable surf-riding equilibria. That is why the continuation method is very useful, because direct time-domain simulations are not capable of capturing unstable equilibria. 
The stability of regions of the equilibrium curve was analyzed by examining the eigenvalues. The inset plot shows eigenvalues in the complex plane for a point on the curve at which the Hopf bifurcation (Figure 4) can be expected. Further development has led to the inclusion of hydrodynamic memory effects into the continuation analysis (Spyrou and Tigkas 2011).

\subsection{The Theory and Code}

The analysis carried out by Spyrou et al. (2009) demonstrated that the LAMP-simulated behavior is consistent with the current theory of broaching-to and surf-riding. It also confirmed the generic nature of the phenomena that had been identified independently and for a very different configuration in earlier research. In particular, the consideration of all six degrees does not change the qualitative picture of the phenomenon.

LAMP-based continuation analysis produced results that are consistent with direct simulation of the stable equilibria as well as the results of previous continuation research of a more theoretical nature.

These results lead to the conclusion that the current theory can be used to explain the outcomes of time-domain simulation with advanced hydrodynamic codes and that advanced hydrodynamic codes can describe a nonlinear dynamical system.

\section{SPATIAL-TEMPORAL FRAMEWORK FOR SURF-RIDING}

In regular waves, a sliding coordinate system with its origin on the wave crest (or any other point fixed to the wave) allows a space-only consideration for surf-riding, as the next or previous wave in space and time is exactly the same. Figure 6 illustrates this approach; the upper part (Figure 6a) shows the balance of thrust and resistance plotted with the spatial representation of the surging wave force. The intersections of these two lines correspond to surf-riding equilibria, which define the topology of the phase plane shown in the lower part (Figure 6b). As the wave is regular, shifting coordinates in space corresponds to shifting coordinates in time, so only one coordinate - usually space - has to be considered.

In irregular seas, the waves are changing in both space and time. Consider an Earth-fixed coordinate system and imagine Figure 6 slowly changing in space for a fixed instant of time. The picture then changes slightly for the next time instant. As the waves change, surf-riding equilibria (actually quasi-equilibria) appear and disappear at different instances of time and points in space. This qualitative description is illustrated in Figure 7.

This rather qualitative consideration produces two main outcomes:

- Celerity of irregular waves must be defined in order to find the equilibria

- All elements of the mathematical model of surf-riding in irregular waves must be functions of position (x-coordinate) and time. 


\section{CELERITY OF IRREGULAR WAVES}

\subsection{Velocity of a Profile}

The most evident idea for irregular wave celerity was to find characteristic points in the profile, track them in time, and take their velocity as an approximation of wave celerity at these points. Belenky et al. (2012) proposed the tracking of zero-crossing points as illustrated in Figure 8. Each curve in Figure 9 is a spatial wave profile plotted at a time instant. Three zero-crossing points, marked by circles, are tracked. The celerity calculated by this method is generally reasonable, but at times can become negative or jump to a very large value.

To gain a better understanding of the nature of this behavior, simplified cases were created with only two and three components (bi- and tri-chromatic waves), as shown in Figures 9 and 10. The tendency for large peaks can be seen even for two frequencies, and is even more dramatic for three frequencies.

Spyrou et al. (2012) had preferred to track the points of constant wave slope, proposing the concept of instantaneous celerity which can be calculated at the exact ship position at each time step. Figure 11 shows the celerity curves calculated for seaway derived from a JONSWAP spectrum. In a further development, they also proposed calculating a characteristic local celerity value corresponding to the point of maximum wave slope that is found in the vicinity of the ship. All these methods converged to the finding that the peaks in the celerity curve are intrinsic to the problem.

The peaks appear to be related to changes in the local wave features, as when one wave overtakes another. As this occurs, a local wave feature may cease to exist and the tracked points disappear and appear in another place, leading to a "spike" in the velocity of those points. Several candidate wave features were considered as points to track, and the smoothest behavior was found for the point of the maximum wave slope on the forward face. As this point can also be associated with the maximum surging force on a ship, it has a physical meaning with regard to surf-riding.

The calculation of wave celerity by tracking the points of maximum wave slope has been implemented in LAMP. In order to consider oblique and short-crested (multi-directional) seas, the algorithm searches for zeros of the derivative of the wave slope in the ship's direction of travel,

$$
\frac{\partial^{3} \zeta_{W}\left(\xi_{\alpha \max }, t\right)}{\partial \xi^{3}}=0
$$

with the condition that they are on the down-slope:

$$
\frac{\partial^{2} \zeta\left(\xi_{\alpha \max }, t\right)}{\partial \xi^{2}}<0
$$


$\zeta_{W}$ is wave elevation, $\xi$ is a coordinate in the mean direction of travel, and $\xi_{\alpha \max }$ is a position of maximum wave slope angle. $\xi$ is related to this Earth-fixed coordinate system as:

$$
\begin{aligned}
& x=x_{G}(t)+\xi \cos \psi \\
& y=y_{G}(t)+\xi \sin \psi
\end{aligned}
$$

$\left(x_{G}, y_{G}\right)$ is the current position of the center of gravity of the ship and $\psi$ is the mean heading angle with respect to the global frame.

For efficiency, the algorithm computes the elevation and its derivatives on a line of points within a characteristic wave length of the ship, then iterates within intervals containing a maximum slope point. The local maximum slope points are tracked in time and the propagation speed of the point closest to the ship provides a practical celerity.

Figure 12 shows a snapshot of a LAMP simulation for the ONR tumblehome hull form running in long-crested irregular waves. The plot shows the wave profile at that time instance along the ship's travel direction with marks for the points of maximum down slope and elevation (crest). The wave in this case is derived from a Bretschneider spectrum with $H_{s}=7 \mathrm{~m}$ and $T_{m}=12.0 \mathrm{~s}$. A portion of the time history of the ship speed and the local wave celerity is shown in Figure 13.

The comparison of ship speed and local wave celerity clearly shows periods of surf-riding (e.g. $t=380-480)$ and periods where the wave is overtaking the ship (e.g. $t=480-600)$. While the initial wave celerity jump at 60 seconds is an artifact of ramping-up the wave, the "spike" in wave speed at $\mathrm{t}=675$ is a case where the phasing of the wave component produces a very rapid local translation of the point of maximum slope and is related to a "merging" of wave faces. However, other discontinuities in the celerity, such as the one at $\mathrm{t}=530$, are simply cases where the closest point of maximum slope switches from one face to another as the waves overtake the ship.

In general, the problem of celerity in irregular waves and how it can affect ship motion is a very deep problem. Spyrou et al. (2014) provided a comprehensive theoretical analysis with an emphasis on the detection of surf-riding.

\subsection{Celerity Based on Instantaneous Frequency}

Looking for a method capable of producing a smoother celerity curve, Spyrou et al. (2014a) proposed, also, an alternate scheme based on the instantaneous frequency, deriving from an envelope presentation of the wave elevation:

$$
\zeta(t, \xi)=A(t, \xi) \cos (\vartheta(t, \xi))
$$


For a case where the wave elevation $\zeta_{W}$ is presented as typical cosine series, the amplitude $A(t, \xi)$ and phase $\vartheta(t, \xi)$ functions are computed from the wave elevations $\zeta_{W}$ and the Hilbert transform of the wave elevations $H\left(\zeta_{w}\right)$ :

$$
\begin{array}{r}
A(t, \xi)=\sqrt{\zeta_{W}^{2}+\left(H\left(\zeta_{W}\right)\right)^{2}} \\
\vartheta(t, \xi)=\arctan \frac{\zeta_{W}}{H\left(\zeta_{W}\right)} \\
\zeta_{W}(t, \xi)=\sum_{i=1}^{N_{\infty}} a_{i} \cos \left(k_{i} \xi-\omega_{i} t+\varphi_{i}\right) \\
H\left(\zeta_{W}(t, \xi)\right)=\sum_{i=1}^{N_{\infty}} a_{i} \sin \left(k_{i} \xi-\omega_{i} t+\varphi_{i}\right)
\end{array}
$$

$a_{i}, \omega_{l}$, and $k_{i}$ are the amplitude, frequency, and wave number of the $i^{\text {th }}$ component, while $\varphi_{i}$ is a random phase shift.

The instantaneous frequency $\omega_{W}$ and the instantaneous wave number $k_{W}$ are then computed as:

$$
\begin{aligned}
& \omega_{W}(t, \xi)=-\frac{\partial \vartheta(t, \xi)}{\partial t} \\
& k_{W}(t, \xi)=\frac{\partial \vartheta(t, \xi)}{\partial \xi}
\end{aligned}
$$

This leads to the following definition of the wave celerity:

$$
c(t, \xi)=\frac{\omega_{W}(t, \xi)}{k_{W}(t, \xi)}
$$

For numerical calculations, it is convenient to substitute equations $(5,7,8)$ into equation (9) and perform differentiation:

$$
c(t, \xi)=-\frac{\frac{\partial H\left(\zeta_{W}\right)}{\partial t} \zeta_{W}-\frac{\partial \zeta_{W}}{\partial t} H\left(\zeta_{W}\right)}{\frac{\partial H\left(\zeta_{W}\right)}{\partial \xi} \zeta_{W}-\frac{\partial \zeta_{W}}{\partial \xi} H\left(\zeta_{W}\right)}
$$

Equation (10) presents the wave celerity as a function of time and space that can be computed everywhere. The result can be compared against the instantaneous celerity calculated through the tracking method. Spyrou et al. (2014a) shows a series of comparisons between the "wave profile" and "instantaneous frequency" calculation schemes, an example of which is reproduced in Figure 14. The two calculations are generally very similar and both show "spikes" in celerity, 
although there is significant difference at the second spike at $440 \mathrm{~s}$. What causes the "spikes" in formula (10)?

Figure 15 shows the spatial profile of the wave celerity calculated for Sea State 7 (Significant wave height $7.5 \mathrm{~m}$, modal period $15 \mathrm{~s}$ ) using a full-bandwidth Bretschneider spectrum and random phases. At this instant in time, there are four spikes at around $1500 \mathrm{~m}$.

One of the advantages of the present scheme of celerity calculation is that the origin of a spike can be analyzed. Consider the instantaneous wave number, which is the denominator in formula (9):

$k_{W}(t, \xi)=\frac{\partial \vartheta(t, \xi)}{\partial \xi}=\frac{\frac{\partial H\left(\zeta_{W}\right)}{\partial \xi} \zeta_{W}-\frac{\partial \zeta_{W}}{\partial \xi} H\left(\zeta_{W}\right)}{\zeta_{W}^{2}+\left(H\left(\zeta_{W}\right)\right)^{2}}$

The spatial profile of the instantaneous wave number is shown in Figure 16. It has a minimum around $1500 \mathrm{~m}$ and this minimum is negative. The curve of the instantaneous wave number crosses zero twice, which results in spikes in the value of instantaneous wave celerity. Figure 17 plots the spatial profile of wave elevations and shows that the minimum of the wave number and spikes of the celerity occur near the secondary maximum, i.e. the local maximum without crossing the line of calm water.

The secondary maxima and minima are related to the origination of new waves. If the local maximum at $1500 \mathrm{~m}$ raises and crosses the calm water line, the new wave will appear. Appearance of the new wave will lead to appearance of a new point of maximum of the wave slope and new zero-crossing. At the instance the new wave appears, the tracked points will make a finite "jump" in the infinitely small period of time causing a theoretically infinite "spike" in celerity. Limiting the smallest value of the instantaneous wave number will limit the value "spike" and will make the celerity equation (10) into a practical formula for calculation.

\section{SURF-RIDING IN IRREGULAR WAVES}

\subsection{Simple Mathematical Model of Surging and Surf-Riding in Irregular Waves}

A simple model for one-degree-of-freedom nonlinear surging was proposed in Belenky et al. (2011):

$$
\left(M+A_{11}\right) \ddot{\xi}_{G}+R\left(\dot{\xi}_{G}\right)-T\left(\dot{\xi}_{G}, n\right)+F_{X}\left(t, \xi_{G}\right)=0
$$

$M$ is mass of the ship, $A_{11}$ is the longitudinal added mass, $R$ is resistance in calm water, $T$ is the thrust in calm water, $n$ is the propeller rotation rate, $F_{X}$ is the surging component of the FroudeKrylov wave force, and $\xi_{\mathrm{G}}$ is longitudinal position of the center of gravity in the Earth-fixed coordinate system. The dot above the symbol indicates temporal derivative. Following Spyrou (2006), a polynomial approximation for thrust and resistance are used: 


$$
\begin{aligned}
& R(U)=r_{1} U+r_{2} U^{2}+r_{3} U^{3} \\
& T(U, n)=\tau_{1} n^{2}+\tau_{2} n U+\tau_{3} U^{2}
\end{aligned}
$$

As the model is meant, at this stage, to be qualitative, a linear wave-body formulation seems to be appropriate for the case. Therefore:

$$
F_{X}\left(t, \xi_{G}\right)=\sum_{i=1}^{N} A_{X i} \cos \left(k_{i} \xi-\omega_{i} t+\varphi_{i}+\gamma_{i}\right)
$$

As a body-linear formulation is adopted, the amplitude $A_{X i}$ and phase shift $\gamma_{i}$ are available via response amplitude and phase operators:

$$
\begin{gathered}
A_{X i}=a_{i} R A O\left(k_{i}\right) \\
R A O\left(k_{i}\right)=\rho g k_{i}\left(\left(\int_{-0.5 L}^{0.5 L} C\left(x, k_{i}\right) \cos \left(k_{i} x\right) d x\right)^{2}+\left(\int_{-0.5 L}^{0.5 L} C\left(x, k_{i}\right) \sin \left(k_{i} x\right) d x\right)^{2}\right)^{1 / 2} \\
C\left(x, k_{i}\right)=2 \int_{-d}^{0} \exp \left(k_{i} z\right) b(x, z+d) d z
\end{gathered}
$$

Here $x$ and $z$ are measured in the ship-fixed coordinate system (positive forwards of amidships and upward from the baseline), $b(x, z)$ is the molded local half-breadth, and $d$ is the draft amidships. The surging phase shift is expressed as:

$$
\gamma_{i}=\arctan \left(\frac{\int_{-0.5 L}^{0.5 L} C\left(x, k_{i}\right) \sin \left(k_{i} x\right) d x}{\int_{-0.5 L}^{0.5 L} C\left(x, k_{i}\right) \cos \left(k_{i} x\right) d x}\right)
$$

The mathematical model described by equations 12 through 18 is essentially an extended time domain version of the model presented in Spyrou (2006). It is expected to reproduce nonlinear surging and surf-riding, which is illustrated in Figure 18.

\subsection{Identification of Surf-Riding Instances in Irregular Waves}

There are two aspects to the problem of identifying instances of surf-riding. The first is that surf-riding in irregular seas is not visually evident from the ship speed, as was illustrated in Figure 13. Without the wave celerity plotted alongside, an observer sees just a periodic stochastic process. The second aspect is to see how well definition of the wave celerity in irregular seas can explain the observed behavior. In a sense, the identification of surf-riding can be used as qualitative validation of the celerity calculation scheme. 
A very basic example of such identification is described by Belenky et al. (2012), using trichromatic waves and zero-crossing wave celerity (see Figure 10). The time histories of surging speed and celerity are shown in Figure 19. The evolution of the surging speed for the first hundred seconds suggests attraction to a surf-riding equilibrium. But is this really the case?

Figure 20 shows a "spatial snapshot" of the forces (surging force vs. balance of thrust and resistance) superimposed with a spatial profile of the wave and its zero-crossing points. The time instant is $t=150 \mathrm{~s}$. The instantaneous position of the ship is indicated via the $\mathrm{x}$-coordinate of the diamond, while the balance between thrust and resistance is indicated by its y-coordinate. The diamond is located at the intersection of the surging force and the balance of the thrust and resistance at the wave celerity. This means that the ship is in equilibrium and surf-riding is observed.

This simple example shows how wave celerity allows an interpretation of the observed motion and establishes the fact of surf-riding. The example also verifies the physical relevance of the calculated wave celerity. The method of calculating celerity based on maximum wave slope was successfully tested for identification of surf-riding by Spyrou et al. (2012, 2014). Spyrou, et al. (2014a) used both methods to calculate the celerity; see Figure 21.

Both methods of the wave celerity calculation seem to identify surf-riding instance in the same way, which is not surprising considering the similarity in the results of the two methods (Figure 14).

\subsection{Phase Space in Irregular Waves}

The position and type of the equilibria defines the topology of the phase plane, so the evolution of surf-riding equilibria in space and time is a logical starting point for analyzing surfriding in irregular waves. Figure 22 shows the calculated loci of equilibria for the tri-chromatic case described above. The result is remarkably consistent with the notional topology presented in Figure 7.

The accuracy of the evaluation of the equilibrium stability was not always sufficient near the points where the equilibria appeared or disappeared, so the stability status of those point was corrected based on geometric considerations. Correct points are shown as two-color symbols. The inset in Figure 22 shows a close-up where the geometrical correction was applied.

The unsteady motion of the equilibria qualitatively changes the phenomenon. A frame of reference moving with the equilibrium is no longer inertial. Also, the dynamical system cannot stay at the equilibrium position, even if the initial conditions correspond exactly to the equilibrium, because the equilibrium will move away. As an analogy, imagine a small heavy ball in a wine glass that is being moved in a circular motion. The ball will continuously "chase" a quasi-equilibrium point that moves around the inside of the glass. 
The phase plane is also changing with time; strictly speaking, the phase plane by itself does not make sense beyond the "spatial snapshot". The phase trajectory becomes a 3D line in hybrid phase-time coordinates (Kontolefas and Spyrou 2015). Projecting to a plane, the set of trajectories behaves as a non-stationary fluid flow. Spyrou et al. (2014a) describe an application of the concept of a Feature Flow Field (Theisel and Seidel 2003), which addresses the problem of feature tracking in non-stationary flow fields. The Feature Flow Field (FFF) method has been proposed for the tracking of a variety of different local features, including critical points of vector fields (such as, in our case, the surf-riding equilibria). Figure 23 shows an example plot for surf-riding, where "saddle and focus-like" structures are present.

Further developments towards the understanding of this time-dependent phase plane are described in Kontolefas and Spyrou (2015). Here is discussed a combined consideration of the feature flow field concept for tracking surf-riding states with the concept of hyperbolic Lagrangian Coherent Structures which can be seen as the finite-time generalization of the manifolds.

\subsection{Statistics of High-Runs}

The study of surf-riding in irregular waves requires the capability to characterize observations from simulations or model tests. However, the identification of surf-riding events in irregular waves is not trivial, particularly if the wave celerity cannot be calculated, which will generally be the case in a model test. It therefore makes sense to also look at the statistics of significant exceedances of the nominal speed by the surge velocity, also known as "high-runs". Themelis et al. (2015) studied the statistics of high-runs depending on spectrum and sea state.

The idea of high-runs is not new. As pointed out in that work, Grim (1963) had looked into the probabilistic quantification of the occurrence and duration of high-runs ("long run") in a following irregular sea, taking into account the strongly nonlinear character of surge motion when the phenomenon occurs. Themelis et al. (2015) also examined the relation between the high-run occurrences and the instantaneous wave celerity (Figure 24).

The conclusion was that the velocity of the high-run shows good correlation with the mean instantaneous celerity when an error metric combining errors of amplitude and phase is applied.

\subsection{Metric for Likelihood of Surf-Riding}

As described at the beginning of this paper, a central element in the implementation of the split-time method is developing a metric for the likelihood of a rare event which can be evaluated at the occurrence of an intermediate event of some sort. An initial proposal for a metric of the likelihood of surf-riding can be found in Spyrou et al. (2014a) and is illustrated in Figure 25. The idea is to compute a "critical distance" in the phase plane between the ship's state (position and velocity) at up-crossing and a critical state (point in the phase plane) from which the ship would be captured into surf-riding. 
The critical point would lie on the line between the initial position of a ship (state at upcrossing) and the position of the stable quasi-equilibrium at the initial instant. A series of "rare" simulations would be performed for initial conditions corresponding to points along this line, and these simulations would determine the conditions that led to surf-riding.

This metric was initially tested with a slowly changing regular wave for which it is possible to explicitly consider the motion of the equilibria. The critical distance could be calculated, but the point to which the system was attracted and, once the critical point was reached, captured into surf-riding was different from the stable equilibrium at the initial moment.

A method of calculating wave celerity based on instantaneous frequency allowed the metric to be tried in irregular waves. The observed picture was more complex. The acceleration of the equilibria is not small and, as a result, the actual attraction does not occur. Instead, the dynamical system moves around the equilibrium path, similar to the picture on Figures 13 and 24. Figure 26 shows the phase plane computed for a moving frame of reference. The velocity of this frame of reference is constant and corresponds to a velocity of the stable equilibrium at the initial moment.

The main difference is that the dynamical system does not follow the stable equilibrium, even if it was placed exactly in the equilibrium position at the initial moment. Furthermore, the equilibria can move toward each other and disappear, releasing the ship from the surf-riding. This means that the criterion for attraction to equilibrium is not as evident as in the case shown in Figure 25. While it is possible to find the critical point visually, the development of a formal criterion of attraction remains for the future work.

\section{CONCLUSIONS AND FUTURE WORK}

This paper has reviewed work related to surf-riding and broaching-to that has been and continues to be performed under the ONR project entitled "A Probabilistic Procedure for Evaluating the Dynamic Stability and Capsizing of Naval Vessels". The present work focusses on how irregular waves influence the phenomenon. The review addressed three major areas:

- Simulation of surf-riding and broaching-to by advanced hydrodynamic codes and the applicability of nonlinear dynamics tools

- Celerity of irregular waves

- Properties of time-dependent phase space of surging and surf-riding in irregular seas and their characterization.

The results of the studies may be summarized in the following conclusions:

- Surf-riding and broaching-to responses predicted via hybrid hydrodynamic codes are consistent with theoretical results, and the concepts and tools developed from ODE-based analysis can be applied to simulation tools as well

- The introduction of irregular waves leads to qualitative changes in surf-riding and broachingto, including:

o Problem must be considered in space and time 
o Surf-riding equilibria moves in an unsteady (accelerating) manner, and can appear and disappear in time

o Surf-riding modes exist for a limited period of time

0 The system attracted to an equilibrium cannot stay with the equilibrium, but instead moves around it.

Problems to be addressed in the next stage of the research project include:

- Formulation of convergence criteria for the metric of likelihood of surf-riding

- Inclusion of sway and yaw into the simplest mathematical model, and study of the uncontrolled turn in irregular waves

- Formulation of the metric of likelihood of broaching-to and capsizing caused by broachingto.

The solution of these problems is directed toward the development of a procedure for a physics-based statistical extrapolation using a limited data set from nonlinear time-domain numerical simulation. It is envisioned that the procedure will consist of the following steps:

- Prepare an extrapolation data set of simulation data

- Set an intermediate threshold providing a reasonable number (thousands) of upcrossings to be observed

- For each upcrossing, compute a metric of the likelihood of surf-riding, broaching-to, or capsizing due to broaching

- Fit a Generalized Pareto Distribution (GPD) to the metric data and evaluate the estimate of the capsizing rate and its confidence interval from the extrapolation of the metric to the level at which capsizing is inevitable.

\section{ACKNOWLEDGEMENTS}

The work described in this paper has been funded by the Office of Naval Research, under Dr. Patrick Purtell, Dr. Ki-Han Kim, and Dr. Thomas Fu, and by ONR Global under Dr. Richard Vogelsong and Dr. Woei-Min Lin. The authors greatly appreciate their support.

Many colleagues have influenced and contributed to our work. The authors would like to acknowledge Prof. Pol Spanos (Rice University); Dr. Art Reed, Mr. Tim Smith, and Mr. Brad Campbell (NSWCCD, David Taylor Model Basin); Dr. Nikos Themelis and Mr. Ioanis Kontolefas (National Technical University of Athens); and Profs. Ross Leadbetter and Vladas Pipiras (University of North Carolina Chapel Hill).

\section{REFERENCES}

Belenky, V.L., Weems, K.M., Lin. W.M., and Spyrou, K.J. 2010, "Numerical Evaluation of Capsizing Probability in Quartering Seas with Split Time Method," Proc. 28th Symp. on Naval Hydrodynamics, Pasadena, California, USA. 
Belenky, V., Spyrou, K., and Weems, K.M. 2011, "Split-Time Method for Surf-Riding and Broaching-To," Proc. 12th Intl. Ship Stability Workshop, Washington D.C., USA, pp. 163168.

Belenky, V., Spyrou, K., and Weems, K. 2012, "Evaluation of the Probability of Surf-Riding in Irregular Waves with the Time-Split Method," Proc. 11th Intl. Conf. on Stability of Ships and Ocean Vehicles STAB 2012, Athens, Greece, pp. 29-37.

Bishop, B., Belknap, W., Turner, C., Simon, B., and Kim, J. 2005, Parametric Investigation on the Influence of GM, Roll Damping, and Above-Water Form on the Roll Response of Model 5613, Report NSWCCD-50-TR-2005/027, Naval Surface Warfare Center/Carderock Division, West Bethesda, Maryland.

Grim, O. 1963, "Surging motion and broaching tendencies in a severe irregular sea," Ocean Dynamics, Vol. 16, No 5, Springer Berlin/Heidelberg, pp. 201-231.

Kontolefas, I, and Spyrou, K.J. 2015, "Coherent Phase-space Structures Governing Surge Dynamics in Astern Seas," Proc. 12th Intl. Conf. on Stability of Ships and Ocean Vehicles (STAB 2015), Glasgow, UK.

Lin, W.M., and Yue, D.K.P. 1990, "Numerical Solutions for Large Amplitude Ship Motions in the Time-Domain," Proc. $18^{\text {th }}$ Symp. on Naval Hydrodynamics, Ann Arbor, Michigan, USA, pp. 41-66.

Lin, W.M., Zhang, S., Weems, K., and Luit, D. 2006, "Numerical simulations of Ship Maneuvering in Waves," Proc. of $26^{\text {th }}$ Symp. on Naval Hydrodynamics, Rome, Italy.

Spyrou, K.J. 1996, "Dynamic instability in quartering seas: The behavior of a ship during broaching," J. of Ship Research, Vol. 40, No 1, pp46-59.

Spyrou, K.J. 1997, "Dynamic instability in quartering seas-Part III: Nonlinear effects on periodic motion," J. of Ship Research, Vol. 41, No 3, pp210-223.

Spyrou, K.J. 2006, "Asymmetric Surging of Ships in Following Seas and its Repercussions for Safety," Nonlinear Dynamics, Vol. 43, pp. 149-172.

Spyrou, K., Weems, K.M., and Belenky V. 2009, "Patterns of Surf-Riding and Broaching-to Captured by Advanced Hydrodynamic Modeling," Proc. 10th Intl. Conf. on Stability of Ships and Ocean Vehicles STAB2009, St. Petersburg, Russia, pp. 331-346.

Spyrou, K., and Tigkas, I. 2011, "Nonlinear Surge Dynamics of a Ship in Astern Seas: "Continuation Analysis" of Periodic States with Hydrodynamic Memory," J. Ship Research, Vol. 55 No 1, pp 19-28.

Spyrou, K., Belenky, V., Themelis, N., and Weems, K. 2012, "Conditions for Surf-riding in an Irregular Seaway," Proc. 11th Intl. Conf. on Stability of Ships and Ocean Vehicles STAB 2012, Athens, Greece, pp. 323-336. 
Spyrou, K.J., Belenky, V., Themelis, N., and Weems, K. 2014, "Detection of Surf-riding Behavior of Ships in Irregular Seas," Nonlinear Dynamics, Vol. 78, No. 1, pp 649-667.

Spyrou, K.J., Belenky, V., Reed, A., Weems, K., Themelis, N., and Kontolefas, I. 2014a, "SplitTime Method for Pure Loss of Stability and Broaching-To," Proc. 30th Symp. Naval Hydrodynamics, Hobart, Tasmania, Australia.

Theisel, H., and Seidel, H. P. 2003, "Feature Flow Fields," Data Visualization 2003. Proc. VisSym 03, pp. 141-148.

Themelis, N., Spyrou, K., and Belenky, V. 2015, "Surf-riding in Multi-chromatic Seas: "Highruns" and the Role of Instantaneous Celerity," Proc. 12th Intl. Conf. on Stability of Ships and Ocean Vehicles (STAB 2015), Glasgow, UK.

Yen, T.-G., Zhang, S., Weems, K., and Lin, W.M. 2010, "Development and Validation of Numerical Simulations for Ship Maneuvering in Calm Water and in Waves," Proc. of $28^{\text {th }}$ Symposium on Naval Hydrodynamics Pasadena, California USA. 


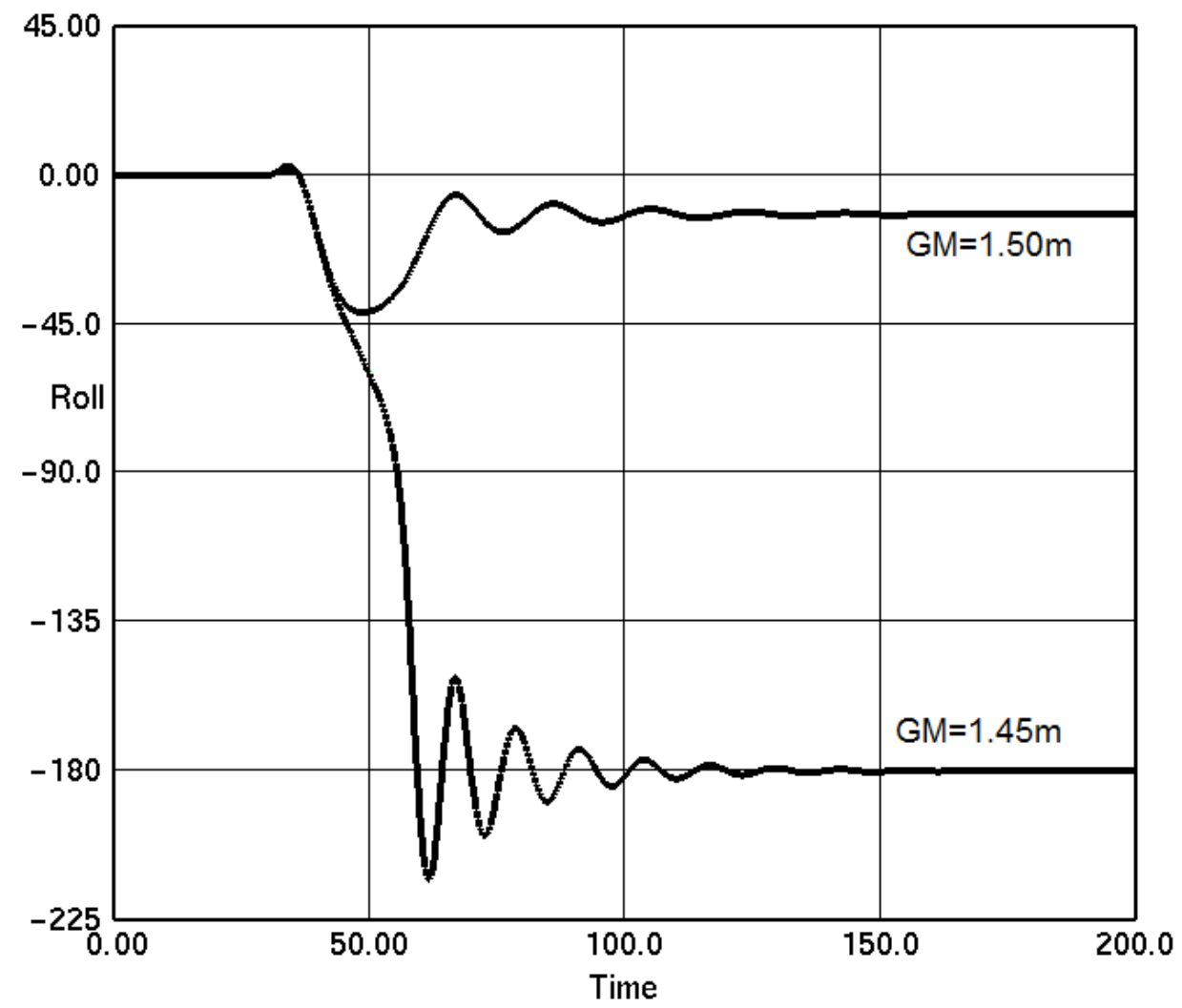

Figure 1: Large heel angle and capsizing due to sharp turn in calm water (Belenky et al. 2010)

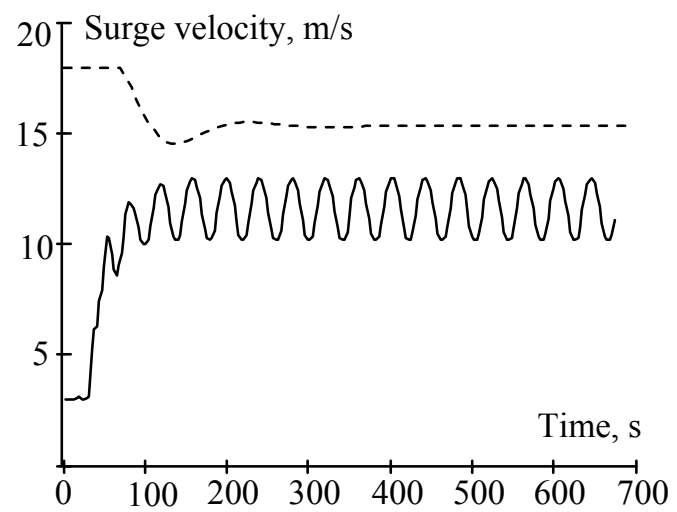

Figure 2: LAMP simulations showing coexistence of surging and surf-riding (Belenky et al. 2010) 
Surf-riding from certain initial conditions

0

$\Delta$ Surf-riding from all initial conditions

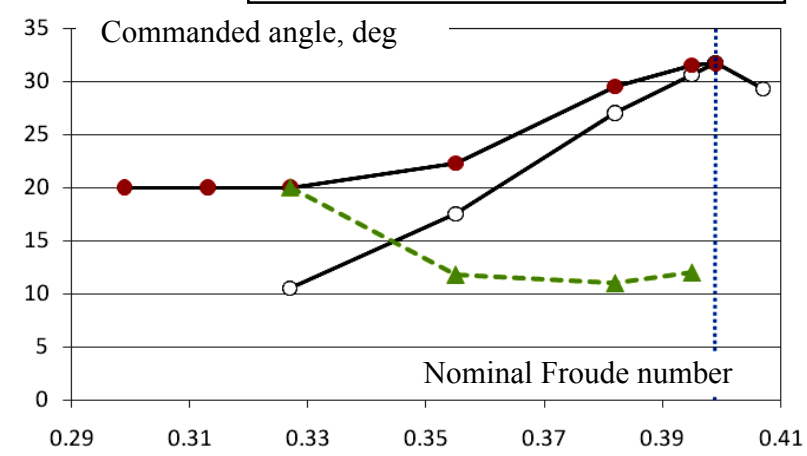

Figure 3: Boundaries of surf-riding in terms of commanded heading as a function of nominal Froude number (Spyrou et al. 2009)

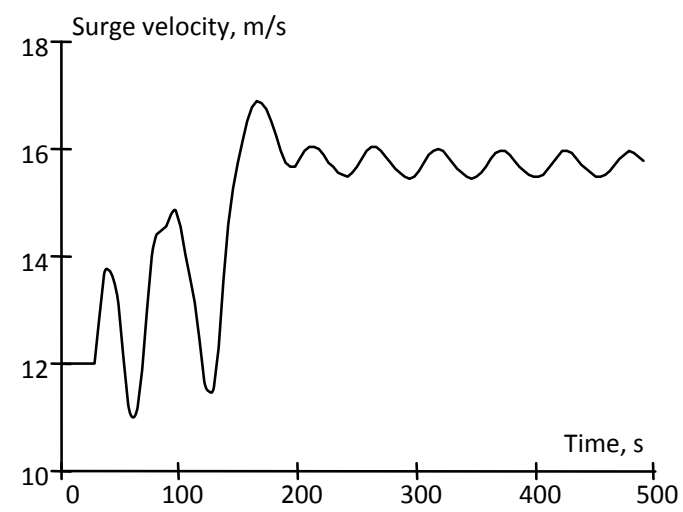

Figure 4: Capture into oscillatory surf-riding for commanded relative heading of $12 \mathrm{deg}$, $\mathrm{Fn}=0.36$; notice the upward jump of mean speed (Spyrou et al. 2009)

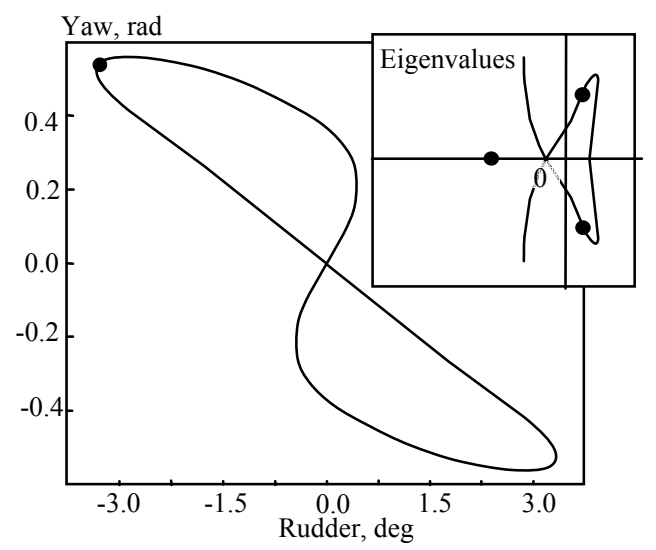

Figure 5: Curve of yaw vs. rudder angle for surf-riding equilibria calculation with DERPAR and LAMP (Belenky et al. 2010) 


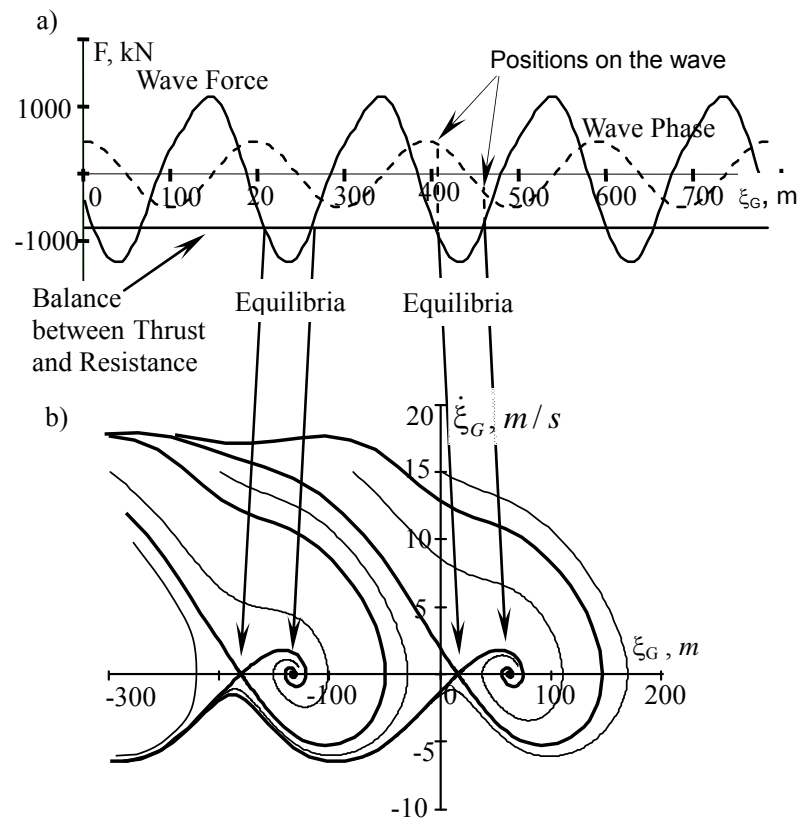

Figure 6: Surf-riding in regular waves - space consideration only: (a) balance of forces and (b) phase plane

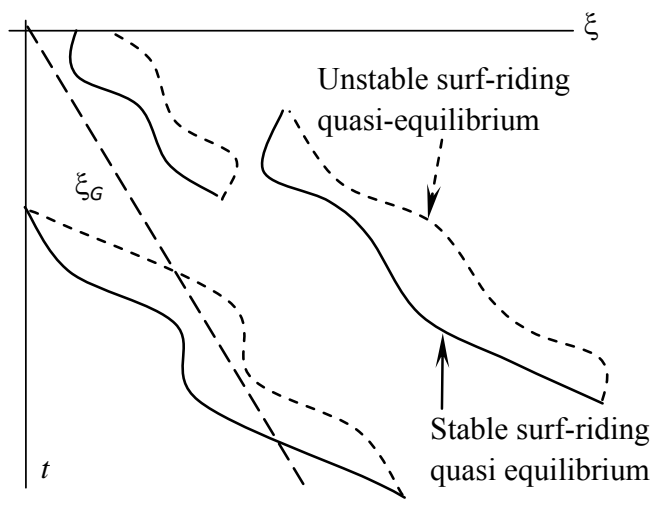

Figure 7: Spatial-temporary framework for surf-riding in irregular seas (Belenky et al. 2011) 


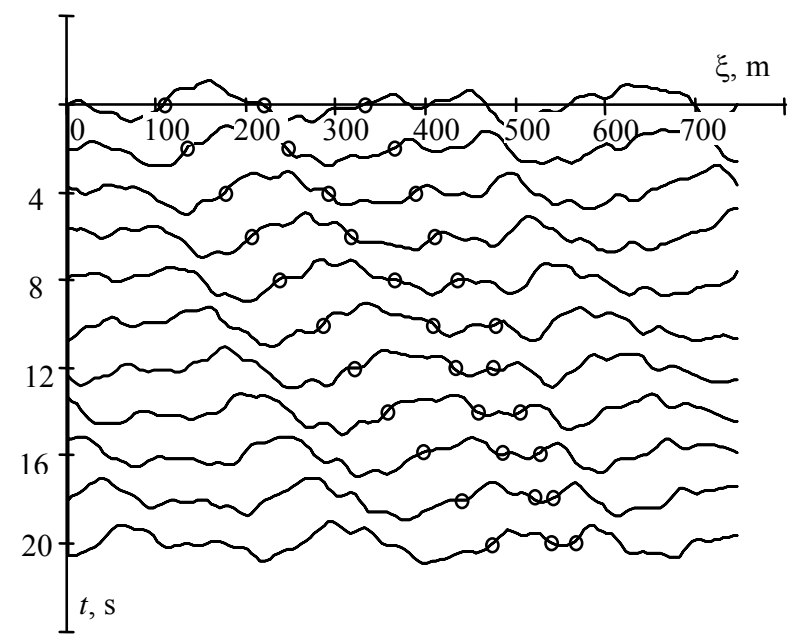

Figure 8: Celerity based on zero-crossing points (Belenky et al. 2012)

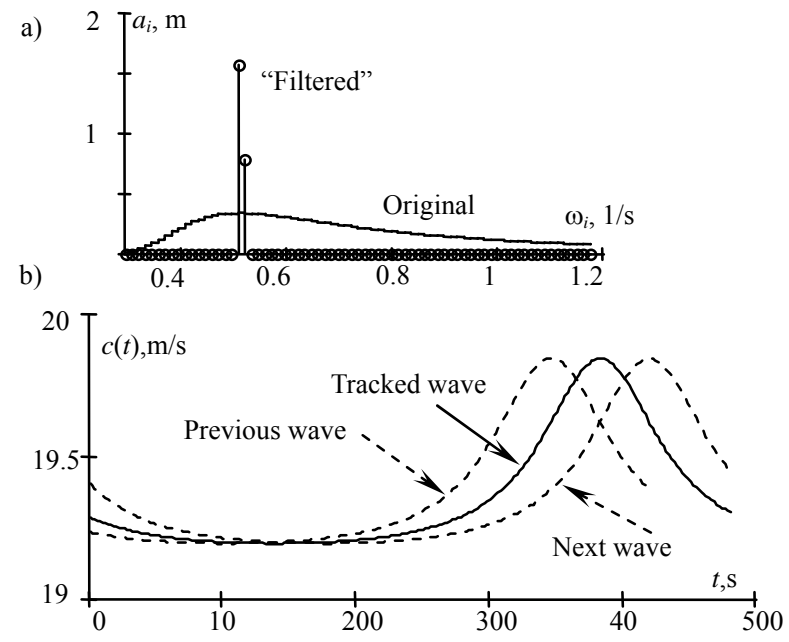

Figure 9: Bi-chromatic waves: (a) spectrum and (b) time history of celerity (Belenky et al. 2012)

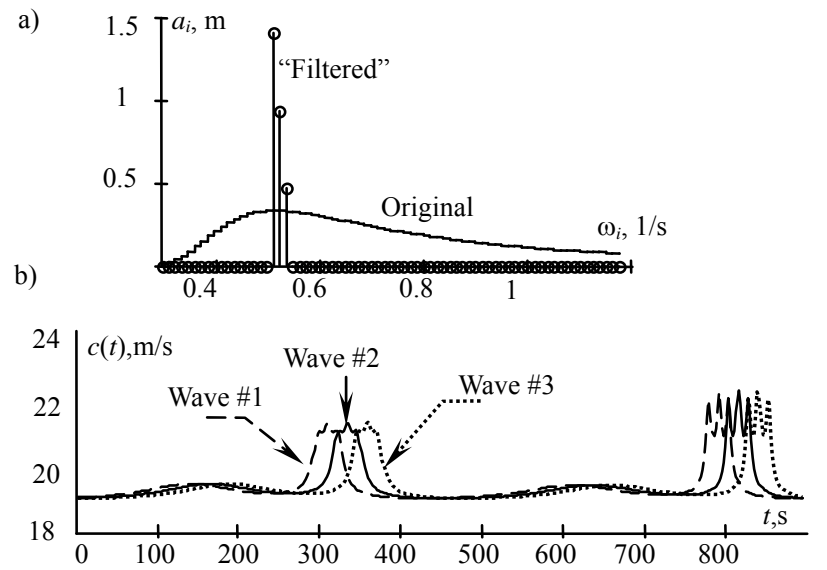

Figure 10: Tri-chromatic waves: (a) spectrum and (b) time history of celerity (Belenky et al. 2012) 


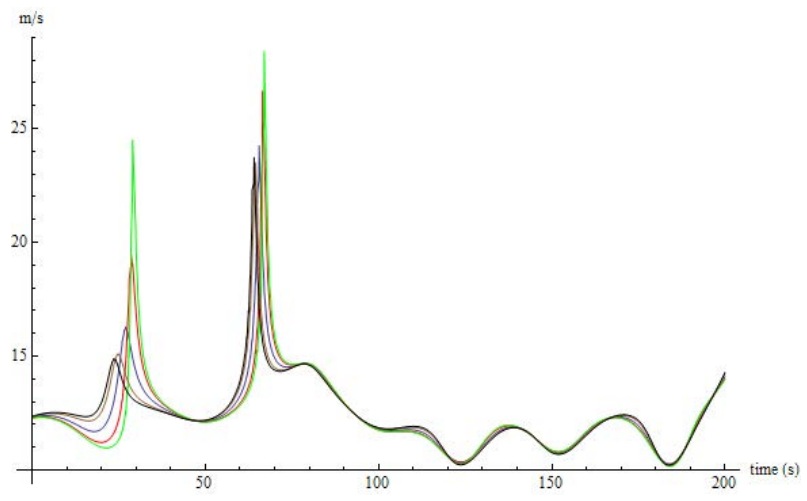

Figure 11: Celerity curves corresponding to wave slopes 1/75, 1/100, 0, -1/100, -1/75 (Spyrou et al. 2012)
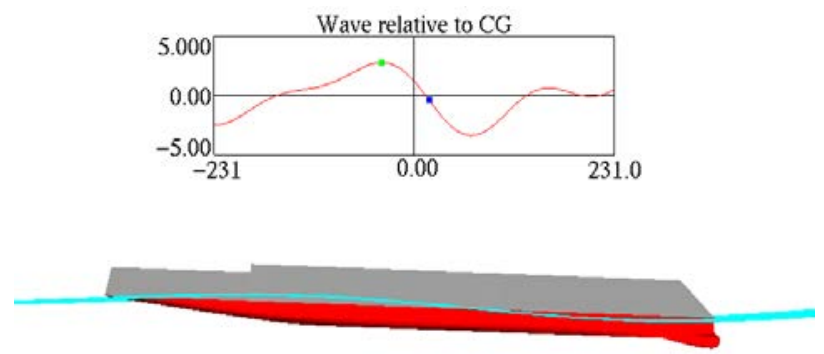

Figure 12: LAMP simulation of ONR Topsides tumblehome hull in irregular following seas (Spyrou et al. 2012)

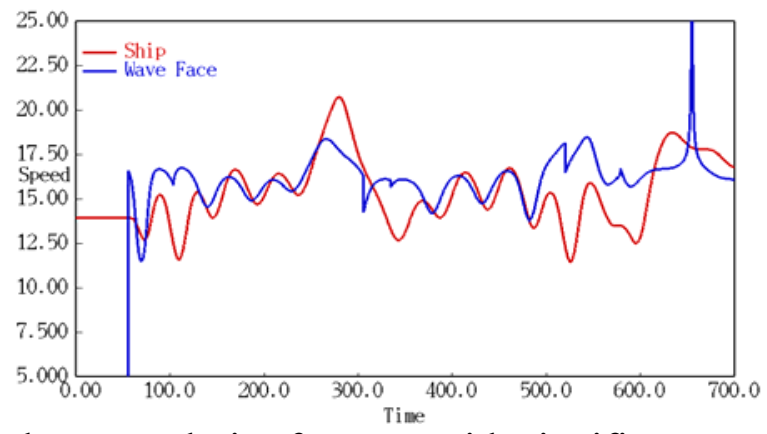

Figure 13: Ship speed and wave celerity for case with significant surf-riding in irregular waves (Spyrou et al. 2012)

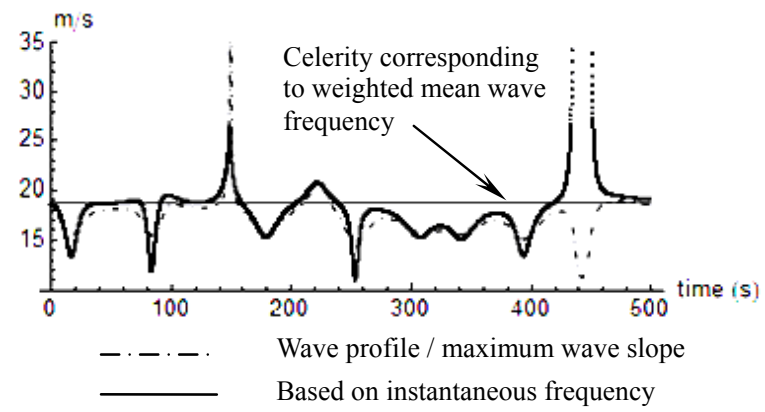

Figure 14: Instantaneous celerity for the bandwidth limited to $20 \%$ of the peak period (Spyrou et al. 2014a) 


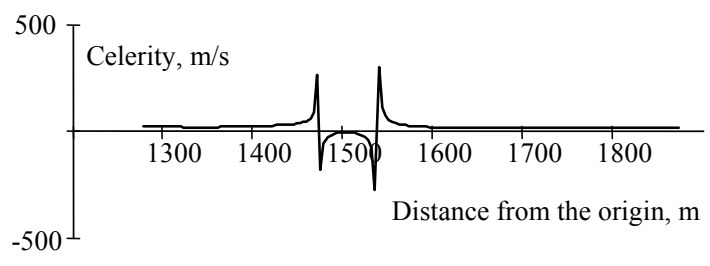

Figure 15: Spatial profile of wave celerity

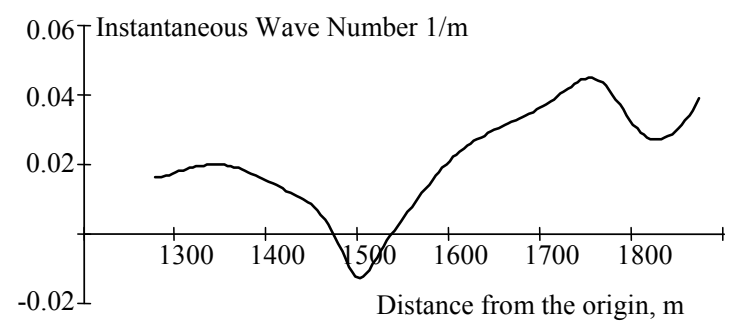

Figure 16: Spatial profile of the instantaneous wave number

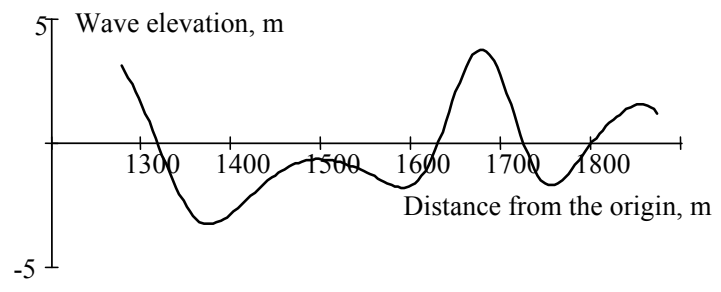

Figure 17: Spatial profile of the wave elevation

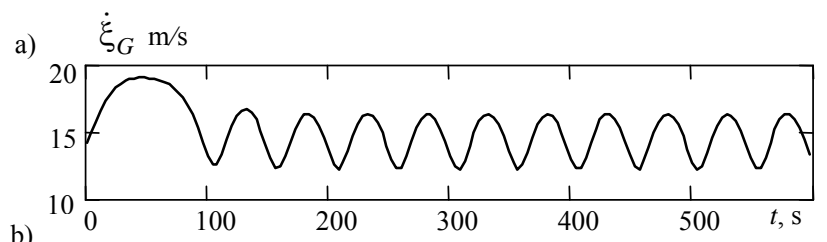

b)

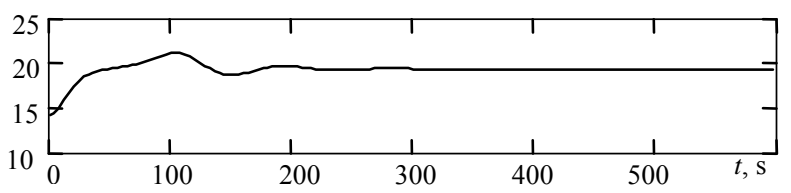

Figure 18: Solution in regular waves: co-existence of (a) surging and (b) surf-riding (Belenky et al. 2011)

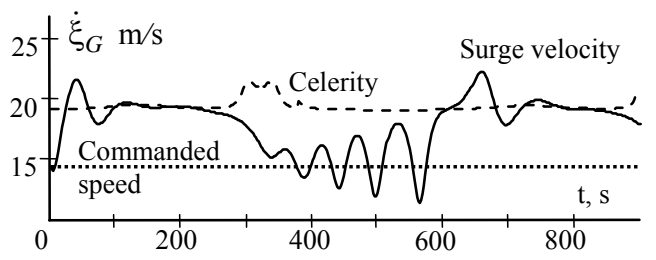

Figure 19: Time histories of surging velocity and celerity for tri-chromatic waves (Belenky et al. 2012) 


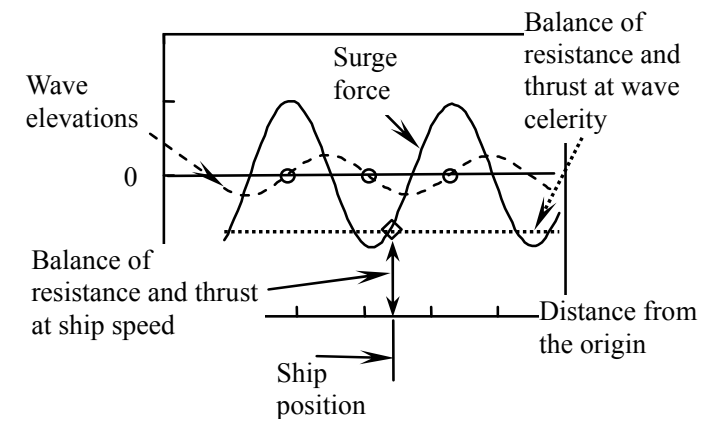

Figure 20: "Spatial snapshot" for $t=150$ s (Belenky et al. 2012)

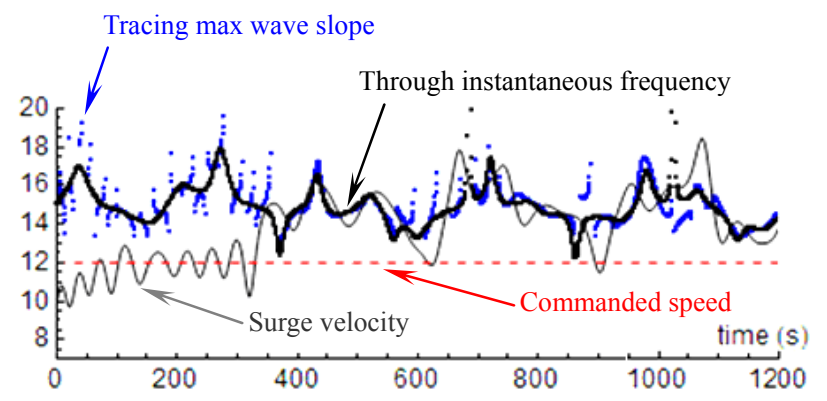

Figure 21: Time histories of surge velocity and celerity calculated with max wave slope and instantaneous frequency (Spyrou et al. 2014a)

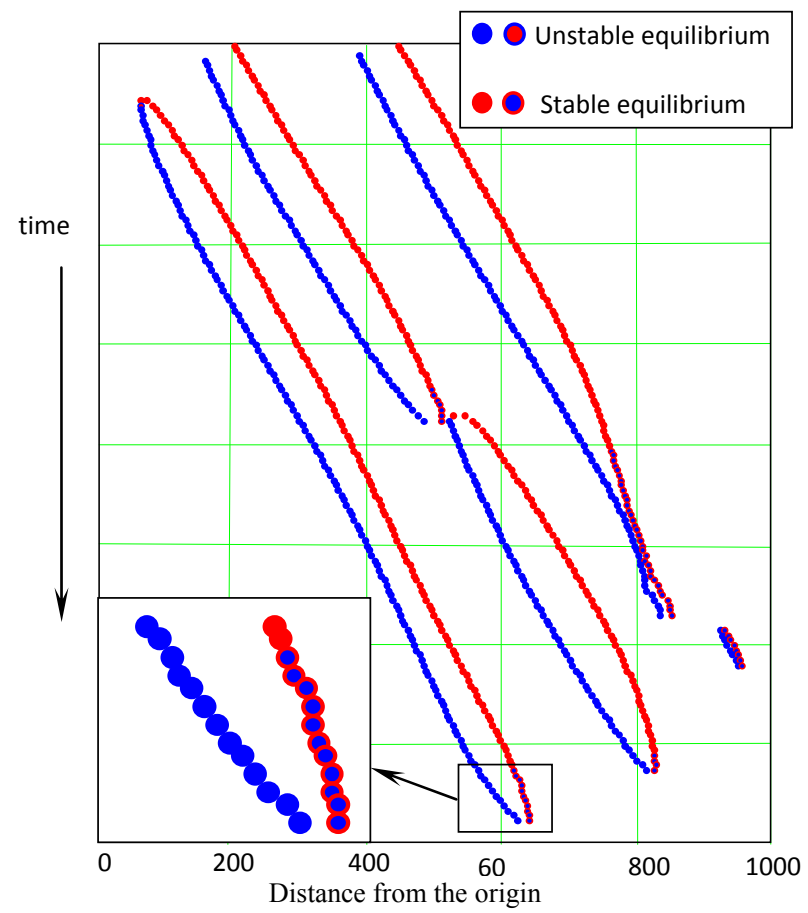

Figure 22: Calculated traces of equilibria 


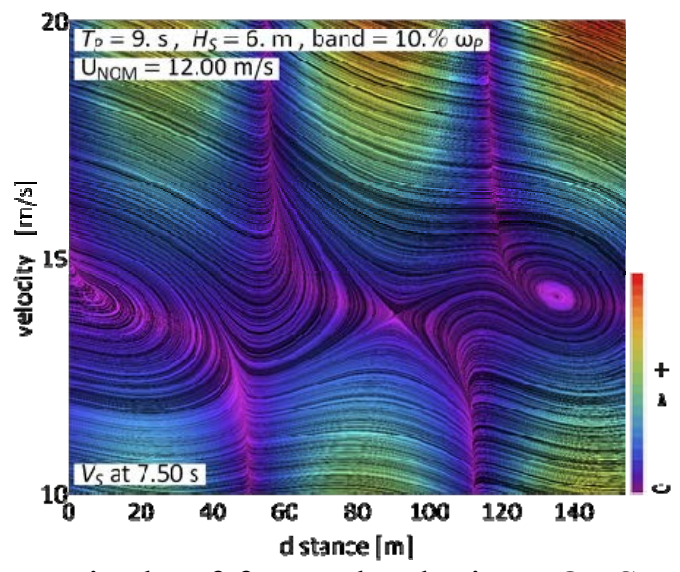

Figure 23: Streamlines and magnitude of forward velocity; JONSWAP Spectrum (Spyrou et al. 2014a)

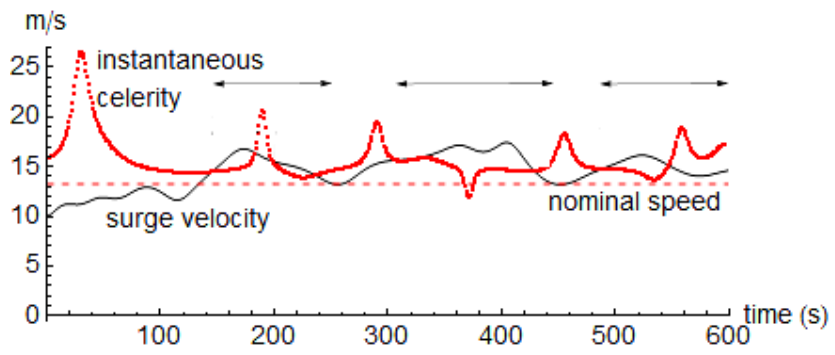

Figure 24: Schematic definition of high-run with the superimposed instantaneous wave celerity (Themelis et al. 2015)

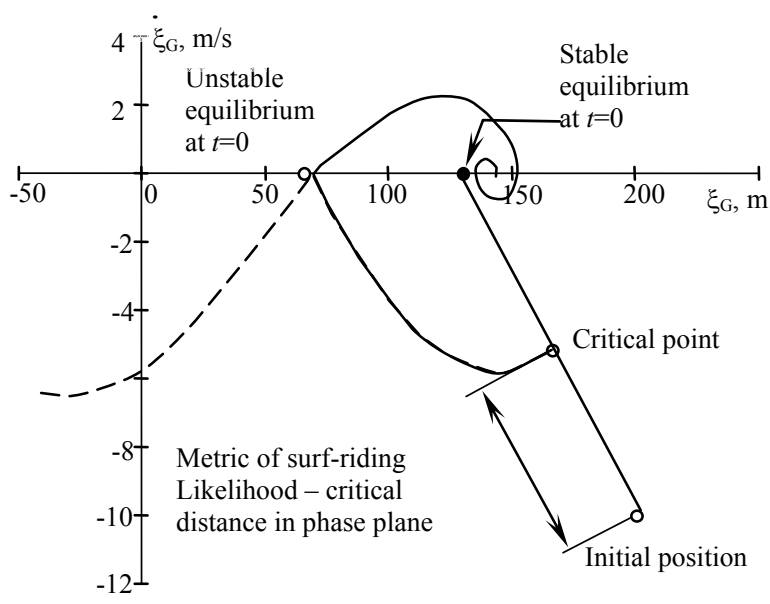

Figure 25: Initial idea for the metric of the likelihood of surf-riding 


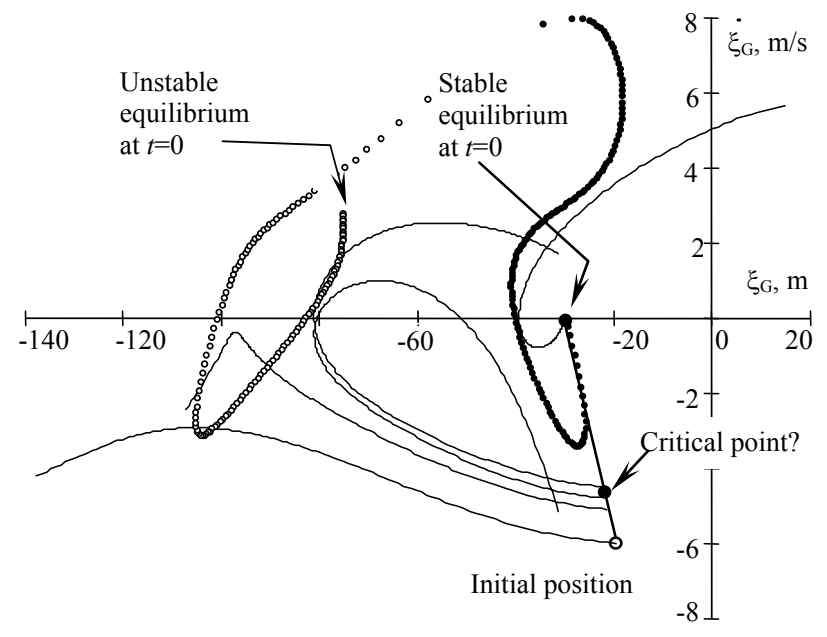

Figure 26: Phase plane trajectories for the critical point determination in calculating a metric for the likelihood of surf-riding in irregular waves 